\title{
The effects of familiarity and previous training on perception of an ambiguous musical figure
}

\author{
BARBARA DAVIDSON, RODERICK P. POWER, and PATRICIA T. MICHIE \\ Macquarie University, Sydney, New South Wales, Australia
}

\begin{abstract}
To study the interaction between familiarity with the "language" in which a musical experience is presented (traditional or contemporary style) and the type of formal training people have had, subjects listened to an ambiguous dichotic figure (Deutsch, 1975) presented under four conditions. In three conditions the figure was preceded by a priming stimulus presented monaurally. In a control condition the dichotic figure was presented three times. Experiment 1 studied responses of nonmusicians, performers of conventional music, and composers of contemporary music. Nonmusicians followed the priming stimulus, performers tended to stream by pitch, and contemporary composers made veridical reports. Experiment 2 examined whether the composers had veridical percepts because of familiarity with the nonlinear language of the dichotic figure or because of an analytic approach to sound perception. Thus Experiment 1 was repeated with nonmusicians, two groups of listeners, and two groups of composers. The nonmusicians followed the priming stimulus, traditional composers and both groups of listeners tended to stream by pitch, and contemporary composers perceived veridically. Implications of the connection between the percepts and familiarity with, or training in, the language of the experience are examined.
\end{abstract}

When we listen to music that involves more than one sound at a time (e.g., a symphony orchestra that has from 2 to 20 separate instrumental parts), what do we perceive? With homophonic ("one voice") music from the classical or romantic periods, the answer is usually that we perceive one tune with a background or accompaniment. With baroque polyphonic ("many voices") music, the answer is more likely to be that we perceive two or more tunes played simultaneously. It is, in fact, not uncommon that a percept of more than one tune results from a solo instrument's playing baroque music (see Figure 1).

How do these different percepts arise? The broad, generalized answer is that the composer organizes the aural context so that either one, two, or more tunes capture the listener's attention. For example, with homophonic music, the tune generally has a complex and often fastmoving rhythm, while the accompaniment has a slower and repetitive rhythm (the ubiquitous "oom-pah-pah"); thus the instrument (or group of instruments) playing the tune is heard as distinct from the rest of the orchestra that contributes to the blocks of chordal accompaniment. The composer may further emphasize separation of tune from accompaniment by making the tune considerably higher or lower than the accompaniment, by allotting it to an instrument whose timbre is distinctly different from the main body of orchestral sound, or by assigning different dynamics or style of playing to tune and accompaniment (e.g., the tune is played loudly and smoothly, while the accompaniment is played softly in a detached style).

\footnotetext{
We are grateful to Karen Rickard and Pamela Warton for critical comments on a draft of this paper. Requests for reprints should be addressed to R. P. Power at the School of Behavioural Sciences, Macquarie University, Sydney, New South Wales 2109, Australia.
}

Thus the composer has many techniques for organizing multiple simultaneous sounds so that they will be perceived as one main stream of melody with background noise. For the creation of a percept of more than one stream of sound from one instrumental voice, the common technique is to alternate notes from a high "tune" with notes from a low "tune" at a moderately fast speed (see McAdams \& Bregman, 1979, p. 26, for the suggestion that at 5 notes $/ \mathrm{sec}$, one perceptual stream is heard, whereas at 10 notes/sec, the high notes perceptually segregate from the low notes to form two separate streams). Figure 1a is an example of such alternation from the treble recorder part of J. S. Bach's Brandenburg Concerto no. 2 (bars 28 and 29), and Figure 1b shows the streams into which people commonly segregate the notes.

Polyphonic music, which has more than one instrumental part, must also be presented in a particular way so that the multiple voices are perceived as separate and meaningful rather than as a confused jumble of sounds. Baroque composers seem to have intuitively subscribed to a model of attention that states that we cannot attend to more than one sound at a time. Consequently, when presenting more than one tune, these composers generally keep all but one of the parts relatively still while one part moves. Figure 2 is another example from J. S. Bach: in these two recorder lines from the opening of Brandenburg Concerto no. 4, recorder 1 holds a long note in bars 1 and 2, while recorder 2 plays a fast-moving part. The slow and fast parts are reversed in bars 7 and 8 .

When a composer wishes to have more than one part simultaneously moving or playing a complex tune, the listener is almost always prepared, because the composer first presents each part separately. This techniquepreparing listeners to expect to hear in a combined presen- 
1a. The actual notation

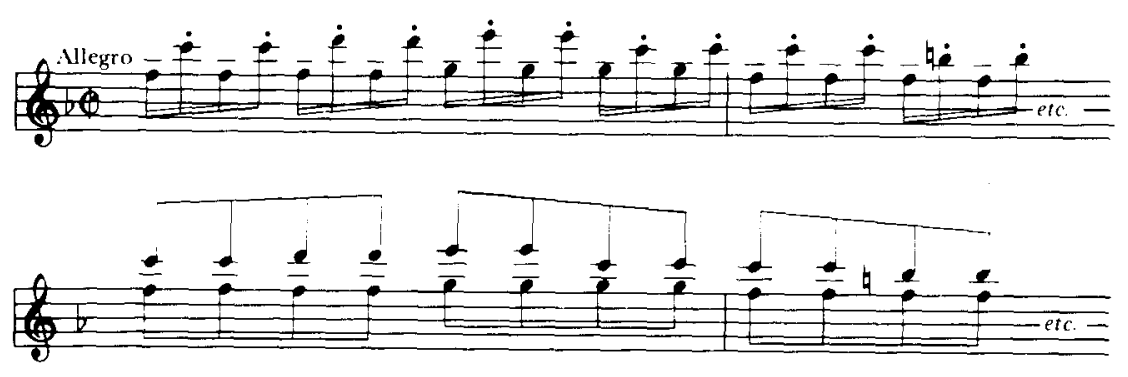

lb. Two perceptual streams

Figure 1. Excerpt from J. S. Bach's Brandenburg Concerto no. 2.

tation two or more tunes that have been presented singlyis at the heart of the great polyphonic musical forms (e.g., fugue, two- and three-part inventions, etc.) and is a subject that we discuss below.

The first of Deutsch's (1974) musical "illusions", illustrates two of the above-mentioned aspects of polyphonic compositional technique: (1) that one has difficulty perceiving two sounds simultaneously, and (2) that one tends to sort high and low sounds into two separate streams. In her experiment, Deutsch presented subjects with alternating high and low Gs; to the right ear, subjects were presented high, low, high, low while to the left ear low, high, low, high (Figure 3). The first point of interest in her results is that not one subject reported that two sounds were played simultaneously. The second is that the mostfavored response was (as shown in Figure 3) of a high $G$ in the right ear followed by a low $G$ in the left ear. Thus it appears that the majority of her subjects determined what they were hearing and where the sound was coming from based on pitch information alone. Deutsch suggested that, since "in everyday life, similar sounds are likely to emanate from the same source and different sounds from different sources"' (Deutsch, 1975, p. 98), we unconsciously assume that similar musical pitches are emanating from one source and different musical pitches from different sources.

Using a more complex stimulus (Figure 4), Deutsch (1975) obtained similar results when she presented sub- jects with ascending and descending $\mathrm{C}$ major scales. The scales were played simultaneously, with notes of each scale switching from ear to ear so that the veridical percept would be as presented in Figure 5a. Seventy percent of her subjects reported hearing the percept shown in Figure 5c; that is, they reported a "wave" pattern percept in which pitch information took precedence over all else, attributing all the high notes to the right ear and all the low notes to the left ear.

Smith, Hausfeld, Power, and Gorta (1982) discovered that replication of Deutsch's findings occurred only when subjects' choices of percepts were limited to the possibilities presented in Figure 5. Moreover, they discovered that musicians were more likely than nonmusicians to report streaming by pitch (suggesting that Deutsch's subjects may have had musical training), and that, when additional information (timbre, loudness, and tune) was given, this was used by subjects so that pitch became only one of many possible cues to correct streaming.

Two of the findings of Smith et al. (1982) have led to the present experiment: first, they reported that their subjects (particularly musicians) considered the veridical percept (Figure 5a) to be a most unlikely tune, and second, they suggested that the musicians' tendency to not report the scale percept (Figure 5b) might have been because they considered this percept a less typical tune than the wave pattern (Figure 5c) (Smith et al., 1982, p. 463). In other words, neither the veridical percept nor the scale

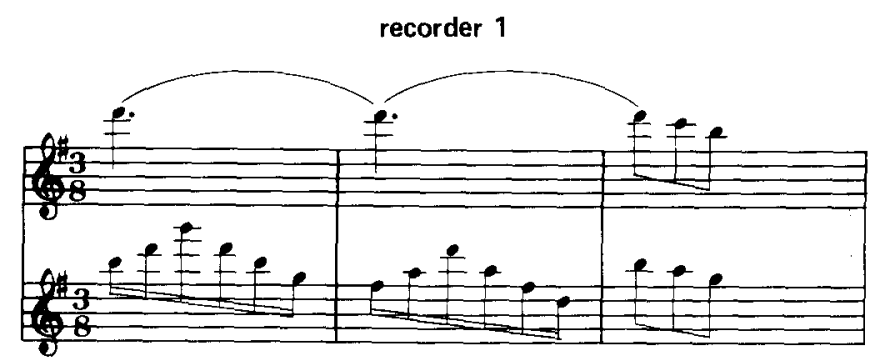

recorder 2

Figure 2. Excerpt from J. S. Bach's Brandenburg Concerto no. 4. 
3a. Stimulus 3b. Most-favored percept

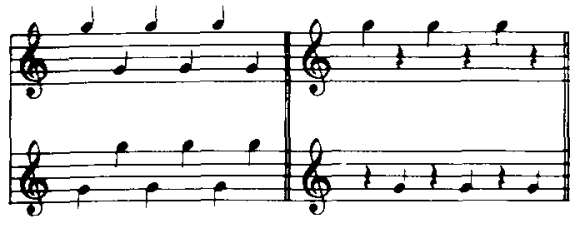

Figure 3. Deutsch's octave illusion. The top stave represents the stimulus and response for the right ear, and the bottom stave represents the same for the left ear.
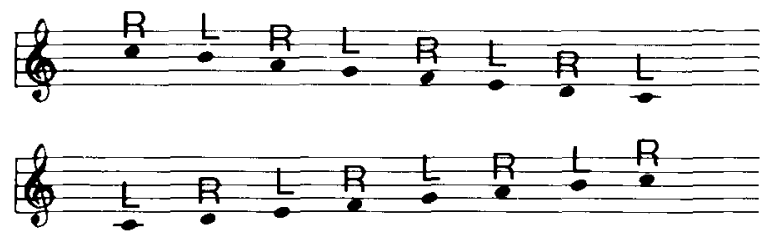

Figure 4. Deutsch's scale illusion. Notes marked $R$ were presented to the right ear, and notes marked $L$ to the left ear.

percept was expected by subjects, which brings us again to the consideration of the baroque technique of presenting tunes separately before interleaving them.

Dowling (1973) found that "when pitch ranges overlap, listeners can overcome interference effects and recognize a familiar target melody if the target is pre-specified, thereby permitting them to search actively for it" ' (p. 322, emphasis added). It seems clear that baroque composers, by presenting tunes separately, are prespecifying them, and leading the listener to search actively for them when they are interleaved. Thus it is reasonable to expect that perception of Deutsch's scale illusion can be influenced by presenting possible percepts (Figure 5) to each ear separately, before presenting the dichotic figure.

We further hypothesized that since musicians with any formal training are familiar with baroque music and its techniques, they will be more likely than nonmusicians to perceive the dichotic figure as being whatever is presented initially and monaurally.

To follow to its limits the hypothesis that experience determines expectations, which determine percepts (an operationalization of Meyer's [1956] theory of expectancy), we chose a third group of subjects, musicians who are currently studying contemporary composition. Because such composition eschews the conventional ideas of "melody"2 and deals with what the uninitiated perceive to be mere noises, we hypothesized that its composers would not find the veridical percept of Deutsch's illusion (Figure 5a) to be as unlikely as would either nonmusicians or conventional musicians. Therefore, we hypothesized that this would be the group most likely to perceive veridically.

To state our hypotheses succinctly, we believed (1) that subjects would perceive the dichotic figure to be what is initially presented to them monaurally, (2) that more musicians than nonmusicians would make this judgment, and (3) that a larger percentage of the contemporary compo- sers would perceive Deutsch's figure veridically than would either conventional musicians or nonmusicians when appropriately primed (stimulus 6a).

\section{EXPERIMENT 1}

\section{Method}

Subjects. There were 48 subjects: 16 nonmusicians (undergraduates from Macquarie University, taking part in the experiment to fulfill course requirements), 16 performers (members of a semiprofessional chamber-music ensemble, taking part in the experiment voluntarily), and 16 contemporary composers (volunteers from a group of participants in a seminar on the use of computer-generated music at the Sydney Conservatorium of Music).

Stimuli. Four different independent stimuli were recorded (Figure 6): (a) the equivalent of the veridical perception of Deutsch's figure in the right ear, followed by the equivalent of the veridical perception of Deutsch's figure in the left ear, followed by the dichotic figure; (b) a descending scale passage beginning on $\mathrm{C}^{1}$ in the right ear, followed by an ascending scale passage beginning on middle $C$ in the left ear, followed by Deutsch's dichotic figure; (c) the upper wave pattern in the right ear, followed by the lower wave pattern in the left ear, followed by Deutsch's dichotic figure; and (d) the control stimulus, Deutsch's dichotic figure played three times so that it matched the others in length.

All stimuli were recorded on a Fairlight Computer Musical Instrument, using the synthesized piano sound, with note duration of $240 \mathrm{msec}$ and interval between onset of successive notes of 250 msec (after Smith et al., 1982).

Each stimulus was re-recorded three times without a break onto a cassette tape to approximate in length Deutsch's 10 repetitions of the dichotic figure.

Design. Our design was a 3 (group: nonmusicians, performers, and composers) $\times 4$ (stimuli: primes and stimuli in Figure 6) factorial, with repeated measures on the last factor. The dependent variables were subjects' choice of two of the six percepts from Figure 5 (one for each ear), after hearing each tape.

Apparatus. For the performers and composers, a set of stereo headphones was attached to a stereo cassette recorder, and subjects were tested individually. The apparatus used for nonmusicians was that used by Smith et al. (1982), and subjects were tested in groups of between 2 and 4 at a time.
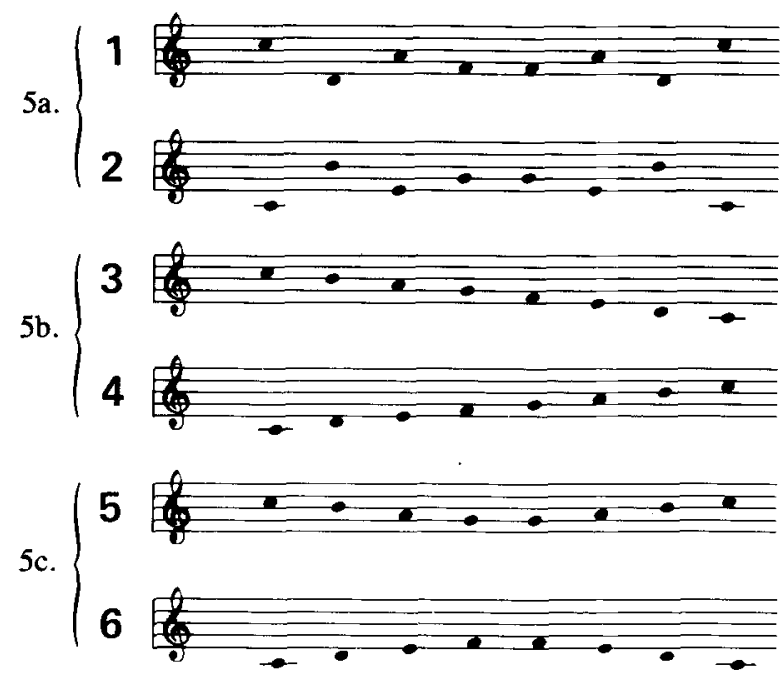

Figure 5. Six possible aural interpretations arising from Deutsch's scale illusion. 
A
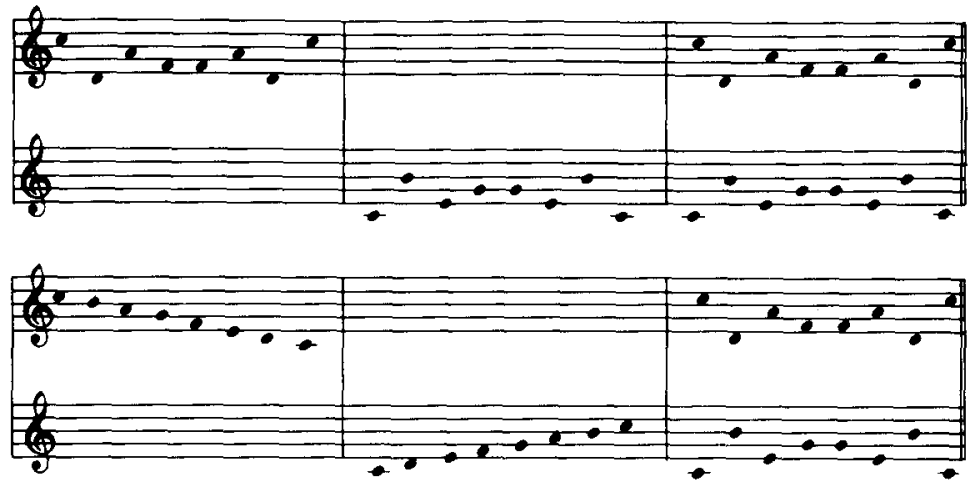

C
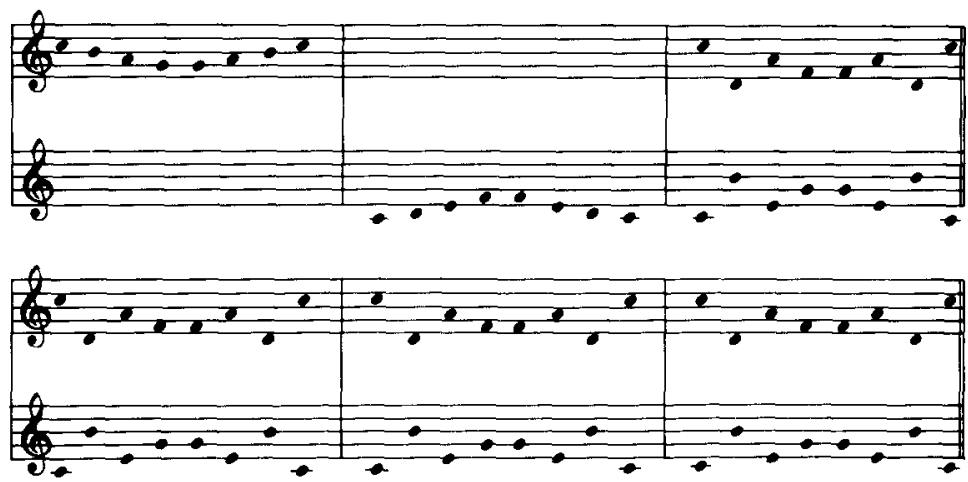

Figure 6. The stimuli presented in Experiments 1 and 2. In each case the first two bars are the priming stimuli, and the last bar is the stimulus on which the subjects reported. In each pair of staves, the upper is presented to the right ear and the lower to the left.

Procedure. Subjects were told that on three of the four tapes they would hear 8 notes in the right ear, then 8 notes in the left ear, then 8 notes presented to both ears at once, and on the other tape they would hear 24 notes played to both ears at once.

They were asked to state which musical pattern they had heard during the last 8 notes on each tape. They chose one number (1-6 in Figure 5) to show which pattern they had heard in their right ear, and one number to show the pattern they heard in the left ear. The experimenters had previously made sure that the nonmusicians understood the basic principles of musical notation (high notes are written high on the stave, low notes written low, etc.). No attempt was made to make the subjects familiar with the possible alternatives, since Smith et al. (1982) found that this simply confused nonmusicians.

Subjects were presented with the four tapes in random order. They listened to a tape and replayed it if they so desired. (Smith et al. [1982] found that subjects never took this option but that having it put them at ease.) They then indicated their perceptions of the dichotic figure by writing the two numbers from the response-choice sheet, which corresponded to Figure 5. As well as being given the six possible percepts, performers and composers were provided with blank music-manuscript paper on which to record any percept that was not covered by the given choices. In our experience, nonmusicians make no use of such opportunity.

\section{Results}

As we predicted, most nonmusicians $(75 \%)$ perceived the dichotic figure to be whatever had been presented to them as a priming stimulus, but this did not hold true, as we predicted it would, for our other two groups. Our second prediction, that more musicians than nonmusicians would be affected by the priming stimulus, received absolutely no support: under all stimulus conditions, most performers $(68 \%)$ perceived either percept $c$ (Figure $5 c$ ) or a figure unique to their group (basically percept $c$, but with the first three notes of the veridical percept $a$ in the right ear). This latter percept is shown as percept $p$ in Figure 7. Composers also failed to confirm our hypothesis that musically trained subjects would follow the prime. Almost all composers $(86 \%)$ perceived veridically under all stimulus conditions. Although failing to confirm our second hypothesis, this strongly supports our third hypothesis, that more contemporary composers than performers and nonmusicians would perceive veridically, not only, as we predicted, when the veridical percept was presented as a priming stimulus, but under all four stimulus conditions.

\section{Discussion}

The interpretation of our results is somewhat complex, since each subject group responded differently. Thus an explanation for any set of responses needs to cover (1) why the group perceived as it did, and (2) why the other groups perceived differently.

Looking first at the response that always corresponded to the prime (i.e., the choice of our nonmusicians), we could say, as we did in our introduction, that these per- 
cepts were chosen because the primes led subjects to $e x$ pect them. Alternatively, it might be suggested that this was a simple illustration of experimenter effects (Orne, 1962). Anecdotal evidence lends some support to the idea that the latter suggestion may not be correct, since most nonmusicians expressed surprise when, in their debriefing, they were told that the dichotic figure had not changed but had been the same on all the stimulus tapes. On the other hand, the fact that in the control condition, when there was no priming stimulus, nonmusicians chose an idiosyncratic mixture of tunes suggests that they were confused by the dichotic figure.

However, whether we say that nonmusicians actually followed the prime or that they perceived a hopeless jumble of sound and simply reported the percept that they believed to be the most likely response (the prime providing them with an answer for which they did not have to search too far), we have to explain why our other two groups did not follow the prime.

Marcel (1984) suggests that the aim of conscious perception is to explain as much of the stimulus input as is possible. To do this, he says, we match the input against a number of "preconscious hypotheses," and consciousness occurs when a fit is made between the input and the hypothesis that best explains it. It seems reasonable to suggest that people with musical training (our performers and composers) would have a wide variety of preconscious hypotheses from which to choose when needing to explain complex structured sound. Nonmusicians, on the other hand, would have few such cognitive constructs and consequently need to rely on hypotheses suggested by the prime.

Marcel's (1984) concept of preconscious hypotheses also provides us with an explanation for the finding that composers of contemporary music were the only group to perceive veridically in all stimulus conditions: their familiarity with nonlinear, nonharmonic sound structures may mean that they have preconscious hypotheses derived from existing schemata (Bartlett, 1932) or frames (Minsky, 1975), which could accommodate the awkward, angular lines of Deutsch's ambiguous figure. (See Shepard and Jordan [1984] for evidence that the Western diatonic scale forms a schema relative to which subjects interpret nondiatonic intervals.) It could also be suggested that our composers' schemata/frames are the same as those of our performers, but that the composers are not as bound by the cognitive constructs because of their professionally necessary analytic approach to sound. It may well be that composers of conventional music would also perceive the ambiguous figure veridically because all composers approach music on two levels: one at which they recognize the overall effect created ("effect" corresponding to the preconscious cognitive construct), and another, atomistic, level at which they ask such questions as "which particular notes, organized in what particular ways are creating the effect I hear?' In other words, composers may be conscious of the whole and of its parts. Thus they are not restricted by holistic preconscious constructs.
The percept chosen by the large proportion of our performers (percept $p$ ) is perhaps the most difficult to explain. We should first note that it is largely made up of percept $c$, the wave pattern on which Deutsch (1975) based her contention that the most important cue to streaming is pitch. We have already noted that pitch separation is indeed one of the most frequently used devices for clear presentation of melodies in classical music. It is not surprising, therefore, that musicians would form their hypotheses using pitch separation, the cue with which they are most familiar. To the basic wave percept, however, a large number of performers joined the first three notes of the veridical percept in the right ear. Three questions arise: How did they perceive this much "correctly"? Why did they perceive only this much "correctly"? Why did this occur with only the right ear?

The answer to the first question may well be simply that they have received a great amount of training in aural discrimination, and the answer to the second question that, since they had no cognitive construct that could accommodate the veridical figure as a whole, each note had to be processed separately. Working memory could not handle 16 pieces of disconnected information. Thus we suggest that musicians may have processed the figure in the following manner: (1) the first four notes in the right ear as discrete units, (2) the remaining four notes in the right ear "chunked" (Miller, 1956) as an ascending scale passage $\left(\mathrm{G}-\mathrm{C}^{1}\right)$, and (3) the notes in the left ear processed either as one chunk (a wave pattern $\mathrm{C}-\mathrm{G}-\mathrm{C}$ ) or as two chunks (ascending scale passage $\mathrm{C}-\mathrm{G}$ and descending scale passage $\mathrm{G}-\mathrm{C}$ ). This would make six or seven pieces of information to be processed, well within the $7 \pm 2$ capacity of working memory.

If we propose that performers failed to perceive the entire veridical stimulus because of the limits of processing capacity, we need to explain why the contemporary composers suffered from no such limitation. The simplest explanation is the composers' familiarity with the medium: cognitive constructs derived from constant exposure to contemporary music enabled our composers to perceive the dichotic figure as a whole (i.e., as one chunk). However, a case for the analytic approach could be made by proposing that, when analyzing the parts that contribute to a musical effect, composers do not necessarily think in linear terms (which would require processing 16 discrete units), but instead search for patterns. Close examination of the veridical percept reveals that the last four notes in the right and left ears are mirror images of the first four notes. Thus, even if they do not perceive the figure in a holistic way, composers could deal with it as four chunks of aural pattern.

The third question regarding the performers' unique percept is " Why do they perceive the fragment of the veridical percept in the right ear alone?" We believe that the answer is simply that all our musicians were rightear/left-hemisphere dominant. It has been suggested that the left hemisphere of right-handed subjects is specialized for analytic, sequential processing, whereas the right 
hemisphere is primarily a holistic, parallel processor (Bradshaw \& Nettleton, 1981). Although it has been argued in the past that musical skill is a right-hemisphere function (Gordon, 1974), it is now known that this is true only for nonmusicians. For experienced musicians, music is primarily a left-hemisphere function (Bever \& Chiarello, 1974). Both of these factors, the analytic sequential nature of left-hemisphere processing and the localization of music to the left hemisphere in musicians, could be responsible for the performers' veridical perception of the first three notes of the stimulus in the right ear. The fact that they were unable to perceive veridically for all eight notes in the right ear may be due to capacity limitations, as discussed above.

\section{EXPERIMENT 2}

\section{Method}

To disentangle the effects of familiarity and analytic training, we repeated Experiment 1 with traditional and contemporary composers and traditional and contemporary listeners, as well as with a group of nonmusicians.

The familiarity hypothesis would predict that frequent exposure to a "language" provides cognitive constructs that enable perception of new material that is presented in the same language, thus predicting that contemporary composers and contemporary listeners would perceive Deutsch's figure veridically. The analytic approach hypothesis would predict that the veridical perception of complex ambiguous stimuli requires specialist training, thus predicting that the two groups of composers would perceive the dichotic figure veridically.

Subjects were 20 nonmusicians (undergraduates from Macquarie University, taking part in the experiment to fulfill course requirements), 20 traditional listeners (volunteers from subscribers to a series of chamber-music concerts), 20 contemporary listeners (volunteers from an audience attending a concert of contemporary piano music), 20 traditional composers (volunteer students from the Sydney Conservatorium of Music), and 20 contemporary composers (also volunteer students from the Sydney Conservatorium of Music). ${ }^{3}$

Criteria for the two listener groups were: (1) that they attended at least five concerts of traditional or contemporary music each year, (2) that they had records or tapes of traditional or contemporary music in their personal music collections, and (3) that they had no more than 4 years of musical training. It would clearly have been desirable for Criterion 3 to have been no musical training at all, but, since this applied to no locatable contemporary listeners, it was necessary to raise the training-level criterion for both groups.

The two composer groups were students in their 3rd or 4th year of tertiary training at the Sydney Conservatorium of Music. Contemporary composers were students who were majoring in contemporary composition, whereas traditional composers were students who were majoring in jazz, a major that involved composing in traditional styles.

\section{Results}

The results are shown in Figure 7. As in Experiment 1, nonmusicians tended to follow the prime, with $75 \%$ reporting percept a following prime $\mathrm{A}, 70 \%$ reporting percept $b$ following prime $B$, and $80 \%$ reporting percept $c$ following prime $\mathrm{C}$. In the control condition $50 \%$ reported an idiosyncratic percept, whereas the remaining $50 \%$ were spread fairly evenly among reports of percepts $a, b$, and $c$.
The percepts of traditional and contemporary listeners and traditional composers did not differ greatly from those reported by the performers in Experiment 1 (i.e., $87 \%$ reported either percept $c$ or percept $p$ in all conditions). There were two differences: one was that a slightly larger percentage of contemporary listeners than of traditional listeners reported the veridical percept, and the other was that a larger number of traditional composers than of contemporary listeners reported the veridical percept.

Contemporary composers once again demonstrated superior perceptual abilities, with $85 \%, 80 \%, 85 \%$, and $80 \%$ reporting the veridical percept in conditions $\mathrm{A}, \mathrm{B}$, $\mathrm{C}$, and $\mathrm{D}$, respectively.

\section{GENERAL DISCUSSION}

This study confirms the previous finding (Smith et al., 1982) that Deutsch's (1975) figure should be regarded as an ambiguous stimulus, at least for people not trained as composers of contemporary music. These composers have stable and veridical percepts (Power, 1977; Power \& Day, 1973; Power, Hausfeld, \& Gorta, 1981) of the stimulus, whereas the experienced listeners and traditional composers in our study had either illusory (i.e., stable nonveridical) or near-illusory percepts. The nonmusicians had fluctuating percepts, which had no independently determinate form and were led by the prime. Deutsch herself (1974) confirmed our view, since she pointed out that her listeners reported fluctuations with repeated listening.

Neither the analytic approach nor the familiarity hypothesis was upheld in a pure form, the results suggesting that both may be necessary to explain the superior performance of contemporary composers. That more contemporary listeners reported the veridical percept than did traditional listeners suggests that familiarity with the language plays some part in enabling veridical perception. The fact that more traditional composers reported the veridical percept than did contemporary listeners suggests, however, that analytic training may be an even more important feature of veridical perception. The differences between the groups, however, were too slight to allow us at this stage to be confident of these suggestions.

We have no hesitation, however, in concluding from the contemporary composers' results that familiarity and analytic training are both necessary for veridical perception of the stimuli. One possible interpretation is that analytic training does not form cognitive constructs as much as it breaks down the constructs we already have, enabling us to perceive outside the limits imposed by schema derived from our everyday experience. Familiarity with the language of a new experience then allows for perception of the experience in "meaningful" chunks.

If the latter conclusion is accurate, it would seem that, in the experiencing of music, familiarity is the most important variable, for it is constant exposure to a language that enables one to recognize the meaningful stimulus patterns or schema implicit in the language. This is presumably all that is necessary for understanding the messages 


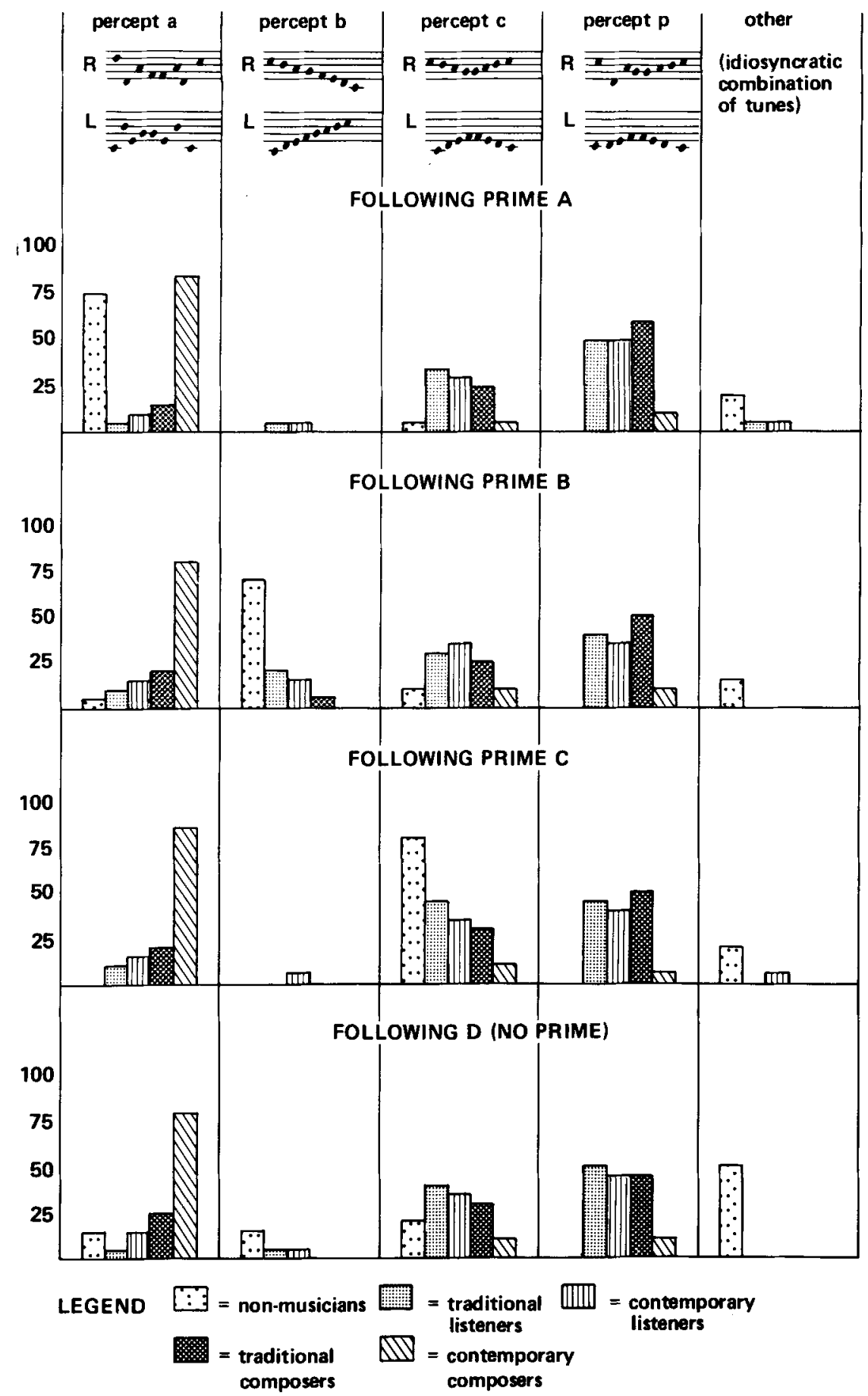

Figure 7. The results of Experiment 2.

that the language conveys. More, however, is necessary for veridical perception at the atomistic level. For one to be able to report accurately on the physical properties of the individual stimuli that contribute to the meaningful patterns, it would appear that one needs to have training that involves analysis and manipulation of the individual stimuli.
We are not proposing that, when familiarity provides meaningful schema and the imposition of those schema bias against veridical perception of individual stimuli, one's experience is not valid. There are many instances in the arts when it might be, in fact, desirable to allow experience of meaning to take precedence over veridical perception of individual stimuli. (There would be little 
joy to be gleaned, for instance, from the perception of a Pointalist painting as a collection of disconnected colored dots.) The important point made by the present study is that, although lack of familiarity with a language makes it difficult to perceive the language in meaningful chunks, familiarity (without analytic training) may make it difficult to perceive accurately the physical properties of stimuli that contribute to meaningful experience.

\section{REFERENCES}

BARTlett, F. C. (1932). Remembering: A study in experimental and social psychology. Cambridge: Cambridge University Press.

Bever, T. G., \& Chiarello, R. J. (1974). Cerebral dominance in musicians and non-musicians. Science, 185, 137-139.

Bradshaw, J. L., \& Nettleton, N. C. (1981). The nature of hemispheric specialization in man. Behavioral \& Brain Sciences, 4, 51-91.

Deutsch, D. (1974). An auditory illusion. Nature, 251, 307-309.

DeUTsCH, D. (1975). Two-channel listening to musical scales. Journal of the Acoustical Society of America, 57, 1156-1160.

Dowling, W. J. (1973). The perception of interleaved melodies. Cognitive Psychology, 5, 322-337.

Dowling, W. J., \& HaRwood, D. L. (1986). Music cognition. New York: Academic Press.

Gordon, H. W. (1974). Auditory specialization of the right and left hemispheres. In M. Kinsbourne \& W. L. Smith (Eds.), Hemisphere disconnection and cerebral function (pp. 126-136). Springfield, IL: Thomas.

MarCel, A. J. (1984). Conscious and unconscious perception: An approach to the relations between phenomenal experience and perceptual processes. Cognitive Psychology, 15, 238-300.

McAdams, S., \& Bregman, A. (1979). Hearing musical streams. Computer Music Journal, 3, 26-44.

MeYer, L. B. (1956). Emotion and meaning in music. Chicago: Chicago University Press.

Miller, G. A. (1956). The magical number seven plus or minus two: Some limits on our capacity for processing information. Psychological Review, 63, 81-97.

MiNSKY, M. (1975). A framework for representing knowledge. In P. H. Winston (Ed.), The psychology of computer vision (pp. 211-280). New York: McGraw-Hill.

ORNE, M. T. (1962). On the social psychology of the psychological experiment: With particular reference to demand characteristics and their implications. American Psychologist, 17, 776-783.

Power, R. P. (1977). Perception. In J. C. Coleman (Ed.), Introductory psychology (pp. 32-73). London: Routledge \& Kegan Paul.

Power, R. P., \& DAY, R. H. (1973). Constancy and illusion of apparent direction of rotary motion in depth: Tests of a theory. Perception \& Psychophysics, 13, 217-223.

PoWer, R. P., HAUSFELD, S., \& GoRTA, A. (1981). Workshops in perception. London: Routledge \& Kegan Paul.

SHePARD, R. N., \& JoRDAN, D. S. (1984). Auditory illusions demonstrating that tones are assimilated to an internalized musical scale. Science, 226, 1333-1334.

Smith, J., Hausfeld, S., Power, R., \& Gorta, A. (1982). Ambiguous musical figures and auditory streaming. Perception \& Psychophysics, 32, 454-464.

\section{NOTES}

1. We place illusions in quotation marks at its first mention since Smith, Hausfeld, Power, and Gorta (1982) found that the effect, as described by Deutsch, was not robust enough to be an illusion (i.e., a misperception that always occurs, as does the misperception of line length in the Müller-Lyer illusion; Power, Hausfeld, \& Gorta, 1981).

2. Our initial search for a group of subjects to whom Deutsch's (1975) figure would make musical sense led us to consider people who are familiar with non-Western music. On closer examination, however, this idea sprung from an ethnocentric expectation that since neither Deutsch's figure nor non-Western music made sense to Westerners, Deutsch's figure would make sense to non-Westerners. As Dowling and Harwood (1986) explain, most cultures base their musical scale on the octave but differ in the ways in which the octave is divided to form modal scales. A modal scale determines "not only the selection of the pitch of melodies, but also the imposition of a tonal hierarchy that shapes the listener's expectations" (p. 115). To alter the pitch of Deutsch's figure and to impose on it the tonal hierarchy of a non-Western scale would be akin to changing the intonation, pronunciation, and rhythm of a spoken English phrase and to expect Chinese speakers to understand the resultant "double Dutch."

3. It would have been of considerable interest to include a group of baroque composers, but we could find none. If any reader is able to put us in touch with a group whose training concentrates on composition in baroque forms, such as two- and three-part inventions and fugues, we would be delighted. We predict that they would perceive veridically, since their training involves developing skills in manipulating two or more equally important melodic strains at once. Thus their percept might be more like the contemporary composers', but for a different reason. 